\title{
The prevalence of clinical signs of ankle instability in club rugby players
}

\author{
E Mellet, A Stewart \\ Physiotherapy Department, School of Therapeutic Sciences, Faculty of Health Sciences, University of the Witwatersrand, Johannesburg, South Africa \\ E Mellet, MSc (Physiotherapy) \\ A Stewart, $\mathrm{PhD}$
}

Corresponding author: A Stewart (aimee.stewart@wits.ac.za)

\begin{abstract}
Background. Ankle injuries are one of the most common injuries in sport and have a high recurrence rate.
Aim. To determine the prevalence of clinical signs of ankle injuries in club rugby players in South Gauteng.

Methods. Institutional ethical clearance was obtained for the study. Of the 180 players from 9 clubs who were eligible for participation in the study, 76\% ( $n=137)$ were recuited. Informed consent was obtained before players were asked to complete a battery of tests. Each player was asked to complete a demographic questionnaire and the Olerud and Molander questionnaire to determine the prevalence of clinical signs of perceived instability. The prevalence of clinical signs of mechanical instability was determined by the anterior drawer test (ADT) and talar tilt test (TTT). Balance and proprioception were assessed by the Balance Error Scoring System (BESS) and this was used to determine the prevalence of clinical signs of functional instability.

Results. The prevalence of perceived instability was $44 \%$. The prevalence of clinical signs of mechanical ankle instability was $33 \%$. There was an increased prevalence of mechanical instability in players who had a history of previous ankle injuries: ADT left $(p=0.003)$; ADT right $(p=0.01)$; TTT left $(p=0.001)$; TTT right ( $p=0.08)$, both tests positive left $(p=0.001)$ and both tests positive right $(p=0.03)$. The prevalence of clinical signs of functional ankle instability depended on the surface and visual input, and was greater as the challenge or perturbation increased.

Conclusion. There was a high prevalence of clinical signs of ankle instability in club rugby players for perceived, mechanical and functional instability. Those with previously injured ankles were more likely to have unstable ankles.
\end{abstract}

S Afr J SM 2013;25(1):23-27. DOI:10.7196/SAJSM.347

Rugby requires explosive power for jumping, quick changes of direction and changes in pace when running. In addition, players tackle and are tackled in close-contact situations. Ankle injuries are common when the players are involved in these types of activities. ${ }^{[1]}$ The lateral ankle ligament complex is the most vulnerable to injury, with $85-95 \%$ of all injuries to the ankle occurring here. ${ }^{[2]}$

Prospective epidemiological studies suggest that ankle injuries account for $8-20 \%$ of all injuries in rugby: ${ }^{[3]} 10 \%$ of the injuries recorded for New Zealand players in the Super $12 ;^{[4]} 11 \%$ in international Australian players; ${ }^{[5]} 14 \%$ in international players at the 2003 World Cup; and 20\% in Scottish district players. ${ }^{[6]}$

In South Africa a study revealed 55.4 injuries per 1000 player game hours and 4.3 injuries per 1000 player training hours in the Super 12 competition, with 7 (11.3\%) ankle injuries sustained in total. ${ }^{[7]}$ The study underlined that it was the first of its kind in South Africa and that there is a need to collect epidemiological data on rugby injuries to develop appropriate management and prevention programmes.

There are 3 different levels of ankle instability, namely perceived, mechanical and functional, which form a continuum. ${ }^{[8,9]}$ Perceived ankle instability is the subjective self-evaluation of the player with regard to ankle function. Mechanical instability is the increase in accessory movement (arthrokinematic motion that cannot voluntarily be produced, e.g. the glide and roll of the talus in the mortise), which translates into an enlarged neutral zone. Mechanical instability is usually the result of a tear or lengthening of one of the ligamentous structures supporting the joint. Residual mechanical instability leads to functional ankle instability. ${ }^{[4]}$ The patient with functional instability has deficits of ankle control in postural control tasks. This is explained in part by the fact that the somatosensory receptors are disrupted and this generates a decreased motor response to maintain postural equilibrium.

The healing time for ligaments is between 6 weeks and 3 months; however, in a systematic review on ankle injuries it has been shown that, at testing, there are still signs of mechanical laxity and functional instability between 6 months and 1 year after the initial injury. ${ }^{[2]}$ Tendon pathology is described as a continuum which requires treatment at appropriate times along the continuum. Initially, reactive tendinopathy occurs as a result of an acute overload or a blow. This may progress to tendon disrepair or failed healing if the tendon becomes chronically overloaded ${ }^{[10]}$ Finally, degenerative tendinopathy may occur with progression of cell and matric changes and cell death; again, due to overload. ${ }^{[2,10]}$

There is a $27 \%$ recurrence of ankle injuries in rugby ${ }^{[3]}$ and a reported $21 \%$ of days absent due to injury could be related to recurrent ankle injuries. ${ }^{[3]}$ It is evident that previous injury predisposes players to future injury, attributed to continued joint dysfunction, pre-existing anatomical and biomechanical factors, or inadequate rehabilitation. ${ }^{[4,9]}$ 
Residual complaints of the player/athlete include a feeling of weakness or the ankle wanting to 'give way', tenderness on palpation, pain or discomfort with running or jumping activities. ${ }^{[10,11]}$

Most of the literature focuses on elite professional sportsmen and little is known about the game and its injuries at an amateur level. The aim of this study was therefore to determine the prevalence of positive clinical signs for perceived, mechanical and functional ankle instability in club rugby players in South Gauteng, and to compare players with $\mathrm{v}$. those without a previous ankle injury.

\section{Method}

We performed a cross-sectional study among all players from the first team squads of the rugby clubs in the South Gauteng region of the Gauteng Lions Rugby Union first division. Nine of the 10 clubs in the region agreed to participate in the study. Each squad included 20 players ( 15 players in the team and 5 reserves). Of the 180 players eligible for inclusion, 137 (76\%) participated. The remaining 43 players met one or more of the following exclusion criteria:

- previous surgery to the lateral ankle ligament complex or ankle joint

- previous injury of the lower extremity (within 3 months of the tests)

- recently diagnosed concussion (within 1 month of the tests)

- current ear infection, head cold or upper respiratory tract infection.

Ethical clearance was obtained from the Human Research Ethics Committee of the University of the Witwatersrand. Permission was given by the relevant authorities to perform the study and players signed informed consent forms prior to participation in the study.

\section{Tests}

Perceived ankle instability was established by the Olerud and Molander questionnaire, ${ }^{[12]}$ which is an investigative tool to make studies of ankle injury more comparable. The Olerud and Molander questionnaire firstly investigates the patient's clinical signs and symptoms, including pain, stiffness and swelling. The second part of the questionnaire surveys the functional impact of the injury on the participant's ability to function in activities of daily life and sport and whether the participant requires external support in the form of taping or bracing to function.

Mechanical integrity was determined by: (i) anterior drawer test (ADT); ${ }^{[1,3]}$ and (ii) talar tilt test (TTT)/stress inversion test. ${ }^{[1,3]}$ The ADT (transverse plane laxity) assesses the integrity of the anterior talofibular ligament, while the TTT is an effective indicator of injury to the calcaneofibular ligament. Positioning is important to isolate the ankle for testing in the ADT. The ankle should be positioned in $10^{\circ}$ of plantar flexion and the knee at $90^{\circ}$, because the most anterior laxity of the ankle will be achieved in this position to better isolate the capsular and ligamentous structures of the ankle. A positive ADT has a sensitivity of $73 \%$ and a specificity of $97 \% .{ }^{[13]}$ When accompanied by a skin dimple during testing, there is a high correlation of approximately $94 \%$, with rupture of the lateral ligament complex. A positive ADT with pain on palpation and signs of haemorrhage has a sensitivity of $100 \%$ and specificity of $77 \% \cdot{ }^{[13]}$ To determine laxity, the test must be done on the affected and unaffected side and then a comparison must be made.
The TTT determines the amount of inversion of the calcaneus when the tibia is stabilised. This, again, is a comparative test for side-to-side differences. This test is only an adjunct to the ADT and is reported to be less reliable in predicting injury.

Functional instability was determined by the Balance Error Scoring System (BESS). ${ }^{[14]}$ The use of BESS has been suggested in a study comparing different techniques for assessing balance. However, it must be clear that no one standing balance test, whether functional or static, can be used to isolate the ankle joint. ${ }^{[15]}$ The ankle is part of the whole kinetic chain and a deficit at any point in the chain will affect balance and ultimately postural control; this needs to be considered even though players were excluded from this study if they had lower extremity pathology. BESS is reliable and valid in controlled laboratory environments. ${ }^{[15]}$

The test is performed in 3 progressive stance positions with the difficulty rating increased: namely double-leg, single-leg and tandem stance. These are repeated on two different surfaces: firm and foam. The number of errors made by the subject in a period of 20 seconds is counted. If a subject makes any errors the test is positive. Errors include opening the eyes, lifting any part of the foot and stepping out of the stance position. ${ }^{[14]}$

For the ADT in this study the subject was asked to lie supine and the knee was semi-flexed to $40^{\circ}$. This position was achieved with the use of a goniometer to eliminate the stabilising effect of a tight gastrocnemius muscle on the excursion of the joint. The first author was positioned in front of the subject. The one hand stabilised the lower leg while cupping the calcaneus with the other hand. The forearm of the hand cupping the calcaneus supported the foot in $10^{\circ}$ of plantar flexion. The foot position was checked with a goniometer. The subject was instructed to relax and to allow the researcher (EM) to move the ankle. The action performed was an anterior displacement or forward pull of the talus and calcaneus while the other hand stabilised the tibia with a constant force. ${ }^{[16]}$

The ADT was deemed positive if the talus glided or slid anteriorly from under the ankle mortise. In certain cases where an audible 'clunk' was heard, the suspected instability was supported by the indication of talar subluxation which indicates greater excursion of the talus and thus instability.

For the TTT, the patient was positioned supine with the first author sitting facing the patient. The test was performed by holding the calcaneus with one hand while the foot was positioned in the neutral position. The other hand was used to stabilise the lower leg, again around the distal tibiofibular region. The calcaneofibular ligament was palpated with one finger to feel the gapping, if present. The hand stabilising the calcaneus applied an inversion stress by rolling the calcaneus inwards to cause talar tilt. The TTT was deemed positive in the presence of excessive tilting or gapping, or if the patient experienced pain while performing the test. A test is deemed positive if tilting or gapping $>3-5 \mathrm{~mm}$ is recorded. ${ }^{[16]}$ The first author performed the ADT and TTT on all players and was blinded as to their previous injuries.

For the BESS, 2 testing conditions on 2 different surfaces were used: single-leg stance for left and right leg, on a firm surface (stable flat surface) and a foam surface (a foam block). Initially the subject had to maintain balance with his eyes open. The player was then asked to repeat the test with his eyes closed, to remove the focus gained from visual input to control balance. 
Single-leg stance was performed by standing for 20 seconds on one leg with the contra-lateral leg held in $30^{\circ}$ of hip flexion and $90^{\circ}$ of knee flexion (ranges were measured by a goniometer) and the foot held approximately $15 \mathrm{~cm}$ off the ground. The subject was asked to close his eyes and place his hands on his iliac crests, while maintaining the appropriate stance. If the subject fell out of position, he had to return to the position as quickly as possible; when the eyes were closed, the player could open his eyes and keep them open until balance was regained before closing them again. The first author, standing $3 \mathrm{~m}$ away, recorded the number of errors made by each subject during the test. A test was deemed positive if the player made an error; subjects were graded according to the number of errors made during the 20-second period. These tests were performed after the ADT and TTT. Prior to performing the test, the subject was instructed, shown and given an opportunity to practise the stance position.

\section{Data analysis}

The data were imported into Stata Release 10 statistical software for analysis. The prevalence of perceived, mechanical and functional instability was presented as a percentage of the whole sample (Statcorp). A chi-square test was used to compare the difference in clinical signs of mechanical and functional ankle instability between the group who had never had an ankle injury and the group who reported a previous ankle injury. All testing was done at a $p<0.05$ level of significance.

\section{Results}

Table 1 summarises the demographic data of the participants. Age, height and weight distribution in the sample varied from adolescent to early 40s, 60 to $130 \mathrm{~kg}$ and 156 to $204 \mathrm{~cm}$, respectively. Occupations were divided into sedentary (corporate or office-bound) and physical (requiring physical labour as part of their duties). More than $50 \%$ of the sample reported a previous ankle injury.

Forty-four per cent of the players perceived that they had ankle instability (Table 2). Pain (28\%), stiffness (29\%) and swelling (15\%) were the most prevalent clinical signs, and $17 \%$ of players reported that they required some kind of external support (Table 3).

Of the 137 players, $33 \%$ had positive tests for clinical signs of mechanical ankle instability irrespective of the side of injury or ligament injured. When side-to-side differences were considered, the

\section{Table 1. Demographic data of participants $(N=137)$}

\begin{tabular}{ll}
\hline Age $($ years $)$, mean $( \pm \mathrm{SD})$ & $24.0( \pm 4.7)$ \\
Height $(\mathrm{cm})$, mean $( \pm \mathrm{SD})$ & $181.5( \pm 7.0)$ \\
Weight $(\mathrm{kg})$, mean $( \pm \mathrm{SD})$ & $93.6( \pm 14.0)$ \\
Occupation, $n(\%)$ & $71(52)$ \\
$\quad$ Sedentary & $66(48)$ \\
$\quad$ Physical & \\
Player position, $n(\%)$ & $73(53)$ \\
$\quad$ Forward & $64(47)$ \\
$\quad$ Backline & \\
Previous injury, $n(\%)$ & $79(58)$ \\
$\quad$ Yes & $58(42)$ \\
No &
\end{tabular}

left side had a higher percentage of clinical signs compared with the right (Tables 4 and 5).

The more difficult the testing conditions, the higher the prevalence of decreased postural control and functional instability. The highest percentages of functional instability were found with the test performed on an unstable surface with the eyes closed, for the right leg $(98 \%)$ and the left leg (96\%), respectively (Table 6).

There were no differences on BESS when comparing those with a previous injury with those without, except for the test for the left leg on a firm surface with the eyes closed. Again, these results suggest that BESS does not isolate the ankle joint, and the results cannot be related to the ankle joint specifically (Table 7).

Thirteen per cent of players reported that they had never fully recovered (Table 8). Forty-two per cent returned to training and participation in matches after being side-lined for a few weeks; less

Table 2. The prevalence of perceived ankle instability: Olerud and Molander questionnaire $(N=137)$

\begin{tabular}{|c|c|c|}
\hline Narration & $\begin{array}{l}\text { Score }^{\star} \\
\%\end{array}$ & $n(\%)$ \\
\hline No perceived instability & 100 & $77(56)$ \\
\hline Perceived instability & $<95$ & $60(44)$ \\
\hline
\end{tabular}

Table 3. Specific perceived functional limitations $(N=137)$

\begin{tabular}{ll}
\hline Perceived functional limitation & $\boldsymbol{n}(\%)$ \\
\hline Pain & $38(28)$ \\
Stiffness & $40(29)$ \\
Swelling & $20(15)$ \\
Impact on climbing stairs & $16(12)$ \\
Impact on running & $6(4)$ \\
Impact on jumping & $8(6)$ \\
Impact on squatting & $9(7)$ \\
Impact on activities of daily life & $11(8)$ \\
Use of ankle supports & $23(17)$
\end{tabular}

Table 4. Prevalence of clinical signs of mechanical ankle instability $(N=137)$

\begin{tabular}{ll}
\hline Side and positive test & $\begin{array}{l}\text { Prevalence } \\
\boldsymbol{n}(\%)\end{array}$ \\
\hline Left & $31(23)$ \\
ADT & $28(20)$ \\
TTT & $25(18)$ \\
ADT and TTT & \\
Right & $23(17)$ \\
ADT & $23(17)$ \\
TTT & $20(15)$ \\
ADT and TTT & \\
Left and right & $45(33)$ \\
Any positive clinical signs &
\end{tabular}


Table 5. Differences in clinical signs of mechanical ankle instability: those with $(N=79) \mathrm{v}$. those without $(N=58)$ previous ankle injuries

\begin{tabular}{|c|c|c|c|}
\hline \multirow[b]{2}{*}{ Positive mechanical test } & \multicolumn{2}{|c|}{ Previous ankle injury } & \multirow[b]{2}{*}{$p$-value } \\
\hline & $\begin{array}{l}\text { Yes } \\
n(\%)\end{array}$ & $\begin{array}{l}\text { No } \\
n(\%)\end{array}$ & \\
\hline ADT, left & $25(32)$ & $6(10)$ & $0.003^{*}$ \\
\hline $\mathrm{ADT}$, right & $19(24)$ & $4(7)$ & $0.010^{*}$ \\
\hline TTT, left & $24(30)$ & $4(7)$ & $0.001^{*}$ \\
\hline TTT, right & $17(22)$ & $6(10)$ & 0.080 \\
\hline Both tests positive, left & $28(35)$ & $6(10)$ & $0.001^{\star}$ \\
\hline Both tests positive, right & $20(25)$ & $6(10)$ & $0.030^{*}$ \\
\hline $\begin{array}{l}\text { Both tests positive, left and } \\
\text { right }\end{array}$ & $48(61)$ & $5(17)$ & $0.001^{\star}$ \\
\hline
\end{tabular}

Table 6. BESS: Prevalence of positive clinical signs of balance deficits (functional instability ${ }^{*}(N=137)$

\begin{tabular}{|c|c|c|c|}
\hline Side and stance surfa & \multicolumn{3}{|c|}{$\begin{array}{l}\text { Positive signs of balance deficits } \\
\text { (functional instability) } \\
n \text { (\%) }\end{array}$} \\
\hline \multicolumn{4}{|l|}{ Left } \\
\hline Firm, eyes open & \multicolumn{3}{|l|}{$13(10)$} \\
\hline Firm, eyes closed & \multicolumn{3}{|l|}{$88(64)$} \\
\hline Foam, eyes open & \multicolumn{3}{|l|}{$65(48)$} \\
\hline Foam, eyes closed & \multicolumn{3}{|c|}{$132(96)$} \\
\hline \multicolumn{4}{|l|}{ Right } \\
\hline Firm, eyes open & \multicolumn{3}{|l|}{$13(10)$} \\
\hline Firm, eyes closed & \multicolumn{3}{|l|}{$76(56)$} \\
\hline Foam, eyes open & \multicolumn{3}{|l|}{$66(48)$} \\
\hline Foam, eyes closed & \multicolumn{3}{|c|}{$134(98)$} \\
\hline \multicolumn{4}{|c|}{$\begin{array}{l}\text { *There were no differences on BESS comparing those with previous injury with those without, excep } \\
\text { for the test for the left leg on a firm surface with the eyes closed. Again, these results suggest that BESS } \\
\text { does not isolate the ankle joint and the results cannot be related to the ankle joint specifically (Table 7) }\end{array}$} \\
\hline \multicolumn{4}{|c|}{$\begin{array}{l}\text { Table 7. Prevalence of the clinical signs of functional ankle } \\
\text { instability: those with }(N=79) \text { v. those without }(N=58) \text { previous } \\
\text { injury }\end{array}$} \\
\hline \multirow[b]{2}{*}{$\begin{array}{l}\text { Side and } \\
\text { functional test }\end{array}$} & \multicolumn{2}{|c|}{ Previous ankle injury } & \\
\hline & $\begin{array}{l}\text { Yes } \\
n / N(\%)\end{array}$ & $\begin{array}{l}\text { No } \\
n / N(\%)\end{array}$ & $p$-value \\
\hline \multicolumn{4}{|l|}{ Left } \\
\hline Firm, eyes open & 10/13 (77) & $3 / 13(23)$ & 0.16 \\
\hline Firm, eyes closed & $56 / 88(64)$ & $32 / 88(36)$ & $0.04^{\star}$ \\
\hline Foam, eyes open & $36 / 65(55)$ & $29 / 65(45)$ & 0.54 \\
\hline Foam, eyes closed & $76 / 132(58)$ & $56 / 132(42)$ & 0.17 \\
\hline \multicolumn{4}{|l|}{ Right } \\
\hline Firm, eyes open & $9 / 13(69)$ & $4 / 13(3)$ & 0.32 \\
\hline
\end{tabular}

Table 8. Time side-lined from games and recovery time $(N=79)^{*}$

\begin{tabular}{ll}
\hline & $\boldsymbol{n}(\%)$ \\
\hline Time side-lined & \\
Not side-lined & $16(20)$ \\
Days & $16(20)$ \\
Weeks & $33(42)$ \\
Months & $14(18)$ \\
Time taken to recover & \\
Days & $40(51)$ \\
Weeks & $25(32)$ \\
Months & $4(5)$ \\
Never fully recovered & $10(13)$ \\
Time spent on the field & \\
Full game & $72(91)$ \\
Only one half & $5(6)$ \\
Bench only & $2(3)$
\end{tabular}

*Thirteen per cent of players reported that they had never recovered. Forty-two per cent of players returned to training and participation in matches after being side-lined for a few weeks. This is less than the 6 weeks that should be observed for soft tissue healing. Twenty per cent returned within days after the injury.

than the 6-week period that should be observed for soft tissue healing. Twenty per cent returned within days after the injury.

\section{Discussion}

Forty-four per cent of players reported perceived signs of instability. Importantly this was a subjective evaluation by the player of the perceived status of the ankle; it is not inferred that these injuries were sustained as a result of playing rugby. The first section of the Olerud and Molander questionnaire describes the physical signs, including pain (28\%), stiffness (29\%), and swelling (15\%). ${ }^{[12]}$ It is interesting that, despite reports of physical signs of ankle injury, these players were still actively participating in practice sessions and games. This raises the question of whether they may be predisposed to future injury due to inadequate healing time management and rehabilitation. ${ }^{[1]}$ The literature reports an initial healing time of 4 - 6 weeks for orientation, aggregation and arrangement of soft tissue. In this phase, normal function is possible, but the athlete is still vulnerable to re-injury. Over the period of 6 months - 2 years, final tissue changes still take place. ${ }^{[10]}$

The functional limitations reported by the players included 4 problems: running (4\%), jumping (6\%), climbing stairs (12\%), and squatting (7\%). Most of these activities are similar to movements in playing rugby. This may suggest that they should not be participating in games and practice, even though this information was only reported by the participants and not actively tested. Where the translation of a joint is not controlled, there will be long-term negative consequences on tissue structure and degeneration of the ankle and subtalar joint, with the possible onset of early osteoarthritis. If the continuum from acute ankle sprain with mechanical deficit to functional instability and then chronic instability occurs, the ankle with perceived instability may eventually end up categorised as chronic instability, this being a sure precursor for early degenerative or arthritic changes. 
Rehabilitation is key to effective and successful return to sport. Acute management should include anti-inflammatory modalities and exercises to maintain range of motion. Once initial healing has occurred, strength and proprioception have to be addressed, and then a graduated return to sport must be supervised. ${ }^{[2,3,10]}$

The prevalence of mechanical instability in the subjects in this study was slightly higher (33\%) than that reported in the literature for ankle injuries in sport in general (10 - 30\%), and higher than the reported prevalence for ankle injuries in rugby players $(9-15 \%) \cdot{ }^{[3-6]}$ At a national or provincial level, injuries are usually managed by multi-disciplinary teams, which may decrease the prevalence of injury with correct rehabilitation or identification of risk factors and pre-injury intervention. Club rugby players are rarely managed at the club and usually pay for their own treatment. There were far more players with positive ADTs and TTTs among those who reported previous injury than those who had never experienced ankle injuries. This shows that there may be residual mechanical laxity after return to participation in sport, or that the players with some sort of mechanical deficit are more likely to sustain injuries. This asks the question of whether these players return too soon or whether they are not fully rehabilitated when they return to the game.

On a stable surface with decreased visual input, $64 \%$ of the players standing on the left leg and $56 \%$ of the players standing on the right leg were deemed functionally unstable. As soon as a player closed his eyes, even when standing on a stable surface, there were signs of instability. This probably points to the importance of visual input to the central nervous system to control the body in space. Pertubation is applied through the constant change in the foot position on the unstable surface. ${ }^{[14]}$ The test does not include the ability to read the surface or adapt to it during the stance phase and simultaneously allow the other foot to clear the ground and propel the body forward during dynamic movement. It has been shown that players with reported functional instability do take longer to stabilise after contacting the ground in a land from a single-leg jump, which assesses functional control. The more challenging the balance perturbation with progression of the test, the greater the positive signs of instability, as shown by the test for the combination of decreased visual input on an unstable surface where $96 \%$ of players on the left and $98 \%$ on the right showed functional signs of instability. For BESS, the whole kinetic chain must be considered and a deficit anywhere in the chain can affect the results. ${ }^{[13,14]}$

\section{Conclusion}

The results of this study demonstrate that ankle injuries in club rugby players are of concern. Performance may be severely hampered by incomplete healing and insufficient rehabilitation following injury, with specific manifestations of ankle instability. These results demonstrate the need for further research into methods of adequately assessing and dealing with injuries, to ensure a constant flow of talent from the clubs into the upper echelons of national rugby, namely the provincial and national teams. In addition, standardised comprehensive management plans and the compilation of a preseason screening tool should be considered, to detect biomechanical abnormalities that could improve post-injury results and lead to a graduated return to sport for these athletes.

\section{References}

1. Zöch C, Fialka-Moser V, Quittan M. Rehabilitation of ligamentous ankle injuries: A review of recent studies. Br J Sports Med 2003;37:291-295. [http://dx.doi. org/10.1136/bjsm.37.4.291]

2. Geiringer SR. Management of the athletic ankle sprain: From acute injury to rehabilitation. Biomech 1997;4:1-5.

3. Sankey RA, Brooks JH, Kemp SP, Haddad FS. The epidemiology of ankle injuries in professional rugby union players. Am J Sports Med 2008;36(12):2414-2424. [http:// dx.doi.org/10.1177/0363546508322889]

4. Targett SG. Injuries in professional rugby union. Clin J Sports Med 1998;8(4):280285.

5. Bathgate A, Best JP, Craig G, Jamieson B. A prospective study of injuries to elite Australian rugby union players. Br J Sports Med 2002;36:265-269. [http://dx.doi. org/10.1136/bjsm.36.4.265]

6. Garraway WM, Lee AJ, Hutton SJ, Russell EB, Macloed AW. Impact of professionalism on injuires in rugby union players. Br J Sports Med 2000;34:348-351. [http://dx.doi. org/10.1136/bjsm.34.5.348]

7. Holtzhausen LJ, Schwellnus MP, Jakoet I, Pretorius AL. The incidence and nature of injuries in South African rugby players in the Rugby Super 12 competition. S Afr Med J 2006;96(12):1260-1265.

8. Denegar CR, Miller SJ. Can chronic ankle instability be prevented? Rethinking management of later ankle sprains. J Athl Train 2002;37(4):430-435.

9. Santos MJ, Lui W. Possible factors related to functional ankle instability. J Orthop Sports Phys Ther 2008;38(3):150-157.

10. Cook JL, Purdam CR. Is tendon pathology a continuum? A pathology model to explain the clinical presentation of load-induced tendinopathy. Br J Sports Med 2008;43:409-416.

11. Van der Wees PJ, Hendriks EJM, Jansen MJ, Van Beers H, De Bie RA, Dekker J. Adherence to physiotherapy clinical guideline acute ankle injury and determinants of adherence: A cohort study. BioMed Central Mus Dis 2007;8:45. [http://dx.doi. org/10.1186/1471-2474-8-45]

12. Olerud C, Molander H. A scoring scale for symptom evaluation after ankle fracture. Arch Orthop Trauma Surg 1984;103:190-194. [http://dx.doi.org/10.1007/ BF00435553]

13. Van Dijk CN. Management of the sprained ankle. Br J Sports Med 2002;36:83-84. 14. Susco TM,Valovich Mcleod TC, Gansneder BM, Schultz SJ. Balance recovers within 20 minutes after exertion as measured by the Balance Error Scoring System. J Athl Train 2004;39(3):241-246.

15. Kovaleski JE, Norrell PM, Heitman RJ, Hollis JM, Pearsall AW. Knee and ankle position, anterior drawer laxity, and stiffness of the ankle complex. J Athl Train 2008;43(3):242-248. [http://dx.doi.org/10.4085/1062-6030-43.3.242]

16. Trojian TH, McKeag DB. Ankle sprains: Expedient assessment and management. Phys Sports Med 1998;26:1-12.

\section{Appendix I}

A pilot study was performed to establish the clarity and reliability of the Olerud and Molander questionnaire, as well as the clinical tests for mechanical instability.

Inter-rater reliability was tested so that one tester, namely the first author, would be reliable and able to conduct all the tests. Inter-rater reliability was established by using the researcher and an assistant to assess the 2 mechanical tests on 14 players from a team not involved in the main study on the same day in 2 separate testing rooms, so that they were blinded to the results scored by the other. The researcher tested a player, and then the player went to the other room where the assistant tested the player until all 14 players had been tested. The researcher then repeated the tests 4 days later in the same manner, blinded to the initial results, to establish intra-rater reliability. The researcher and the assistant physiotherapist agreed on all subjects for both mechanical tests performed. 